\title{
Estimation of the User's Emotional State by Keystroke Dynamics
}

\author{
Rinky Solanki \\ Institute of Engineering and Technology, DAVV \\ Indore, India
}

\author{
Pragya Shukla \\ Institute of Engineering and Technology, DAVV \\ Indore, India
}

\begin{abstract}
Emotions play a significant role in human computer interaction (HCI). Up to the present, different methods for emotion measurement have been developed like facial expression analysis, voice intonation analysis, thermal imaging etc. Our approach in this paper is to identify users' emotional state based on using standard keyboard. According to the proposed scenarios, emotional states investigated within this research include, but are not limited to, the following ones: confidence, sadness, happiness, tiredness, nervousness, anger.
\end{abstract}

\section{General Terms \\ Human Factors}

\section{Keywords}

Biometrics, keystroke dynamics, emotion recognition, Human computer interaction

\section{INTRODUCTION}

Recognizing emotions of computer users are an increasingly important factor. Traditionally, computer system do not adapt according to user's emotional states. If the computer system were capable of recognizing the emotional state that the user is going through in a particular period of time they would have many benefits for intelligent computers. Various methods have been developed and explored in order to identify emotional state. These methods are based on different resources, i.e. physiological (body temperature, heart rate) [1], visual (facial expression, gestures) [2, 3], audio (speech, voice) [4], input devices (keystroke dynamics, mouse movement, touch screen) $[5,6]$ etc.

In this paper we describe a new behavioral method for measuring user affect with the standard computer input devices keyboard. We are developing an inexpensive, nonintrusive method, which is able to extract user affect while the user is working on the system.

Keystroke dynamics refers to the method of identifying or confirming the identity of an individual based on the manner and the rhythm of typing on a keyboard. Keystroke dynamics is a behavioural biometric, this means that the biometric factor is 'something you do'.A research on identifying emotional states using keystroke dynamics in a real-life application has been presented in [5]

Previously before 1980's, in the Second World War a technique known as The Fist of the Sender was used by military intelligence to distinguish based on the rhythm whether a morse code message was send by ally or enemy. These days everyone has at least one computer keyboard, making keystroke dynamics the easiest biometric solution to implement in terms of hardware.

\section{EMOTIONS AND THEIR MEASUREMENT METHOD}

There are different definitions of emotions, but in this paper we adopt the three components common to all emotions [7]

1. Emotions provoke physiological changes and an emotional expression. They influence our body by changes in heart rate, skin conductivity and similar. This combination is the emotional expression, which can range from shivering (fear) to vomiting (disgust).

2. Emotions create a notion to take action. In this sense they influence our behavior. Anger prepares us to fight, fear prepares us to freeze or flee.

3. Emotions have a meaningful cognitive component.

According to Eysenck [7], affective states can be distinguished by time: emotion is a reaction to stimuli that lasts for seconds or minutes, mood is an emotional state that lasts for hours or days and personality is an inclination to feel certain emotions. In this paper, we use the term 'emotional state' to indicate current (temporary) state of a person irrespective of its origin (stimuli, mood or personality).

\section{Emotion Classification:}

Emotions are common to all humans. All of us feel them in a similar way but possibly with different intensity. Emotion differs from person to person. For the precise scientific use, emotions have to be classified. There are two interesting models of emotion classification: The model of basic emotions and the model of valence/arousal.

\section{a) Basic Emotions:}

This approach applies labels to emotions with some languages or words. They are invoked by universal factors (like reaching/missing a goal) and result in different signals. They are based in the autonomous nervous system (ANS) and therefore provoke similar physiological reactions that can easily be observed already in infants [8]

Anger: A reaction on an obstacle on our path to complete a goal.

Disgust: A reaction on something that does not comply with our moral and social expectations.

Fear: A reaction on the possibility of not reaching a goal.

Happiness: A reaction on the completion of a goal. 
Sadness: A reaction on the definitive knowledge that our goal was not completed.

Surprise: A reaction on an unexpected event.

\section{b) Valence-Arousal Model:}

These features were based on the idea presented by Lang [9], in which he suggested that emotions can be classified in twodimensions (AV space): arousal (intensity of the feeling, the excitement of the person.) and valence (feeling is positive or negative.).

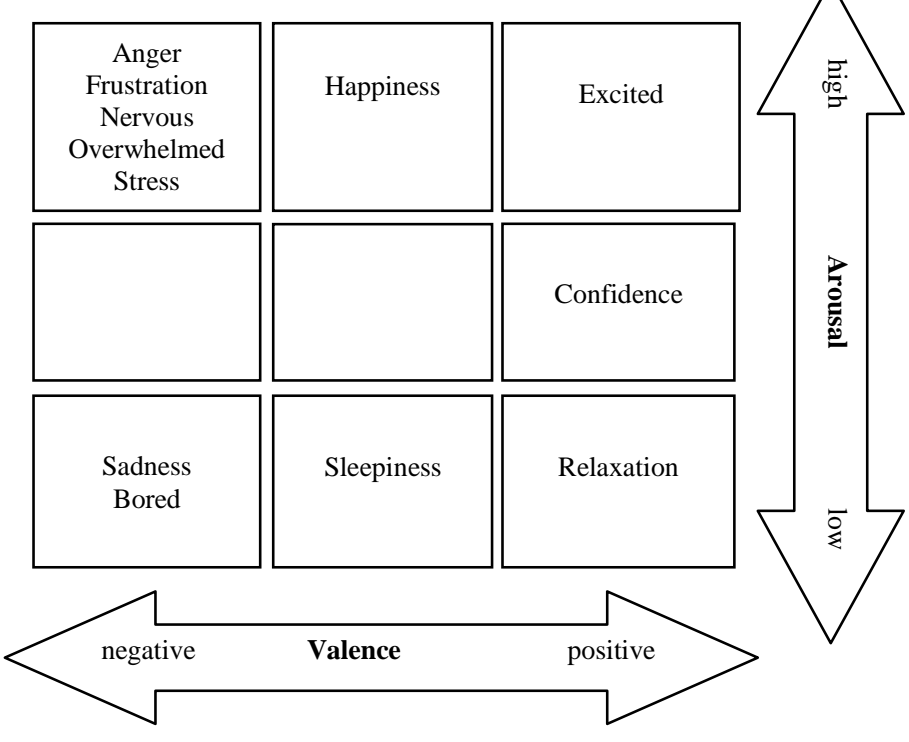

Fig 1: Emotional states in arousal valence space

\section{EMOTION RECOGNITION PROCESS}

Analysis of keystroke dynamics can be broadly classified into two types - static or fixed text and dynamic or free text.

\subsection{Data Collection}

\subsubsection{Fixed text analysis}

Fixed text analysis involves analyzing keystroke behavior of an individual on fixed piece of text from the famous novel "Alice's Adventures in Wonderland" [10].The user are asked to type the paragraph and after that, the user had to choose one of the seven emotional states which best matched with the user's current emotional state. The advantage of this method was no need to induce any emotional states. The user was unable to copying and pasting the fixed text. The user could close the data collection window if they were too busy.

\subsubsection{Free text analysis}

Free text analysis involves continuous or periodic monitoring of keystroke behavior. It is first checked when a user logs in the system and continues thereafter.We use a java application which can run in background to collect keystroke timings (key press time and key release time). The users were interrupted from time to time by recording their recently typed text and asking to choose an emotional state.

\subsection{Feature Extraction:}

After obtaining user's keystroke timing information it is necessary to represent the samples in a way recognizable by the algorithms which are going to be used. We may divide the feature extracted into two groups: timing and frequency parameters $[5,11]$. Timing features are the duration times of single keys and key sequences pressing, flight time and typing speed. Frequency features indicate how often a user presses selected keys, such as backspace, delete, shift, alt etc. A single feature vector is usually created by averaging the parameters' values taken during a predefined period of time.

Keystroke Features: In figure 2, four types of keystroke features are illustrated. We use all these features in our application.

3.2.1. Dwell Time (or Hold Time):It is the time (in milliseconds) between a key press and key release of the same key. In other words, how long a key is pressed until it is released?

Duration of each keystroke $\left(\boldsymbol{D}_{\mathbf{1}}\right)=$

Key release time $\left(\boldsymbol{R}_{\mathbf{1}}\right)-$ Key press time $\left(\boldsymbol{P}_{\mathbf{1}}\right)$

\subsubsection{Flight Time (or Keystroke Latency):}

a) Press - Press: Interval between two successive key presses (always positive).

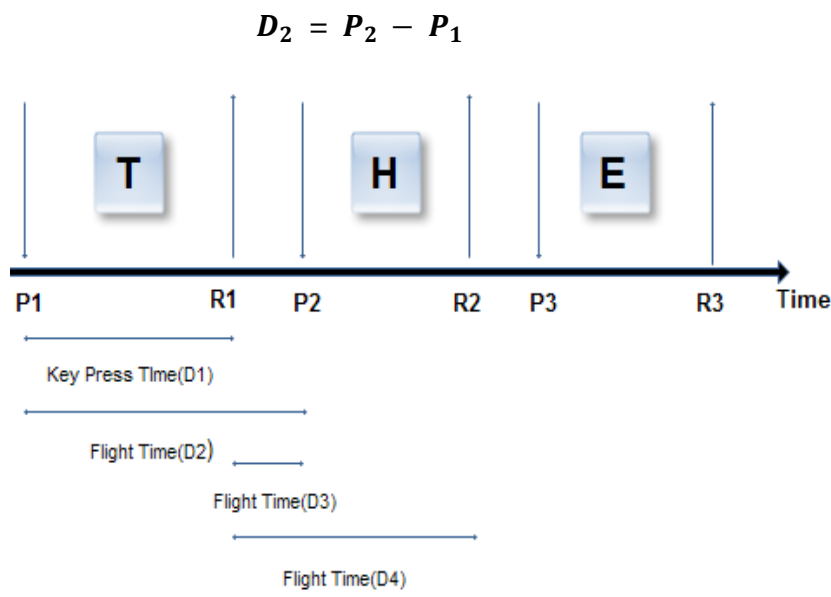

Fig 2: Keystroke features: Dwell Time and Flight Time b) Release - Press: Interval between a key release and the next key press time (may be negative if next key is pressed before previous key is released).

$$
D_{3}=P_{2}-R_{1}
$$

c) Release - Release: Interval between two successive key releases (always positive).

$$
D_{4}=R_{2}-R_{1}
$$

Table 1. Extracted Keystroke Features and their meanings

\begin{tabular}{|l|l|}
\hline $\begin{array}{l}\text { Keystroke } \\
\text { Features }\end{array}$ & Meaning \\
\hline Num_char & Number of characters in the sample \\
\hline Num_mistakes & $\begin{array}{l}\text { Number of mistakes found (backspace + } \\
\text { delete) }\end{array}$ \\
\hline Num_digits & Number of digits found \\
\hline Num_symbols & Number of symbols found \\
\hline Num_letter & Number of letters found \\
\hline
\end{tabular}




\begin{tabular}{|l|l|}
\hline Key_count & Total number of key pressed \\
\hline Key_code & The ASCII code that represents each key \\
\hline Dwell_time & $\begin{array}{l}\text { Time between the key press and key release } \\
\text { of the same key }\end{array}$ \\
\hline Flight_time & $\begin{array}{l}\text { Time duration in between releasing a key } \\
\text { and pressing the next key }\end{array}$ \\
\hline
\end{tabular}

Minimum, Maximum, Mean, Mode, Medium, Standard Deviation, and Variance of dwell time, flight time is extracted instead of extracting a single number. These features were used to build training data set.

\subsection{Creation of training set containing labeled data}

There are two main approaches to emotion labeling. One of the method is to label emotions by a human, the second approach is automatic labeling. There are different types of labels (like excitement, boredom, joy, surprise etc.) where people are able to choose from a predefined list of word labels during questionnaire. The questionnaires may use scales such as the Likert scale presenting a range of responses to each question [12] or Self-AssessmentManikin (SAM) technique, which is a graphical way of expressing valence, arousal and dominance [13] and labelling using a continuous scale that allows to determine emotion gradation in terms of valence, arousal.

In our application, the labels for the training data are assigned according to specially designed questionnaires given to the users for the best accuracy of the trained system.

\subsection{Classifier's learning}

There are various machine learning methods that can be used for classifiers creation in emotion recognition through keystroke dynamics like decision trees [5], Bayesian networks [14], neural networks [16] etc.

Rule based systems for classification have the disadvantage that they involve sharp cutoffs for continuous attributes so we used fuzzy logic as a solution to recognize users' emotional state. Fuzzy logic systems provide graphical tools to assist users in. The created classifier can be used for emotion classification in different applications.

\section{CONCLUSIONS}

The article describes the analysis of user's emotional state via analyzing keystroke timings. If the system knows how its users feel, it can appropriately react to these moods and change its behavior accordingly. The present research facilitates user's emotional by capturing keystroke patterns as well as by analysis of self-reports of each individual user. A training set of user data is captured and processed. The set of data is then compared with the information in the database.

The main benefit of using keystroke dynamics is that the required equipment, any standard keyboard which is inexpensive and already widely used in most computer systems.

\section{ACKNOWLEDGEMENT}

The Author would like to thank her Guide for his constant support and encouragement in her work. The Author would also like to thank college Management for supporting in her research work.

\section{REFERENCES}

[1] Kalakowska A, Zavadskas EK, Seniut M et al (2011), Web-based Biometric Computer Mouse Advisory System to Analyze a User's Emotions and Work Productivity, Engineering Applications of Artificial Intelligence 24: 928-945

[2] Maat L, Pantic M (2007), Gaze-X: Adaptive, Affective, Multimodal Interface for Single-User Office Scenarios, Human Computing, LNAI 4451, Springer-Verlag: 251271

[3] Szwoch M (2013), FEEDB: a Multimodal Database of Facial Expressions and Emotions, Proceedings of the 6th International Conference on Human System Interaction, Gdarisk

[4] Schuller B, Lang M, Rigoll G (2002), Multimodal emotion recognition in audiovisual communication, Proceedings of IEEE International Conference on Multimedia and Expo, ICME 2002, Lausanne

[5] Clayton Epp, Michael Lippold, and Regan L. Mandryk, Identifying Emotional States using Keystroke Dynamics, CHI 2011

[6] Zimmermann P, Gomez P, Danuser B, Schar S (2006), Extending usability: putting affect into the userexperience, Proceedings of the 4th Nordic Conf. on Human-Computer Interaction, Oslo, pp 27-32

[7] T. Dalgleish. Psychology: An Integrated Approach. by Michael W. Eysenck. Longman, Harlow, 1998.

[8] C. Wade and C. Tavris. Psychology. HarperCollins College Publishers, New York, 4 edition, 1996.

[9] Lang, P. 1995. The emotion probe. American Psychologist. 50, 5 (1995), 372-385.

[10] Carroll, L. Alice's Adventures in Wonderland. The Gutenberg Project, 2008.

[11] Althothali A (2011) Modeling user affect using interaction events (thesis), University of Waterloo, Canada

[12] Likert R (1932), A technique for the measurement of attitudes, Archives of Psychology, 22(140): 1-55

[13] Bradley M, Lang P (1994) Measuring emotion: the selfassessment manikin and the semantic differential. Journal of behavior therapy and experimental psychiatry, 25(1):49-59

[14] Lee H, Choi YS, Lee S, Park IP (2012), Towards Unobtrusive Emotion Recognition for Affective Social Communication, Proceedings of the 9th IEEE Consumer Communications and Networking Conference: 260-264

[15] Althothali A (2011) Modeling user affect using interaction events (thesis), University of Waterloo, Canada 